\title{
Evaluation of Weakly Singular Integrals Via Generalized Cartesian Product Rules Based on the Double Exponential Formula
}

\author{
Athanasios G. Polimeridis and Juan R. Mosig, Fellow, IEEE
}

\begin{abstract}
Various weakly singular integrals over triangular and quadrangular domains, arising in the mixed potential integral equation formulations, are computed with the help of novel generalized Cartesian product rules. The proposed integration schemes utilize the so-called double exponential quadrature rule, originally developed for the integration of functions with singularities at the endpoints of the associated integration interval. The final formulas can easily be incorporated in the context of singularity subtraction, singularity cancellation and fully-numerical methods, often used for the evaluation of multidimensional singular integrals. The performed numerical experiments clearly reveal the superior overall performance of the proposed method over the existing numerical integration methods.
\end{abstract}

Index Terms-Double exponential quadrature rule, generalized Cartesian product rules, method of moments, mixed potential integral equations, weakly singular integrals.

\section{INTRODUCTION}

$\mathbf{M}$ IXED potential integral equation formulations have been extensively used over the last years for solving a wide variety of practical electromagnetic radiation and scattering problems [1]-[3]. A typical numerical solution of the mixed potential integral equations using the Rao-Wilton-Glisson basis functions [1] and a Galerkin method of moments approach [2], calls for the computation of 4-D integrals over surface subdomains (triangles, rectangles or general polygons), according to the selected discretization scheme.

The aforementioned multidimensional integrals can be classified as smooth, near-singular and weakly singular (though integrable) integrals, depending on the behavior of the integrand, which is also strongly related to the proximity of the discretized subdomains. To be more specific, the weakly singular cases arise when the two surface elements coincide (coincident integration), share a common edge (edge adjacent integration), or share a common vertex (vertex adjacent integration). Correspondingly, the near-singular integrals arise when the outer and inner elements don't share any common points but their distance is very small, while the smooth integrals cover all the other possible cases.

Manuscript received July 17, 2009; revised November 24, 2009; accepted January 11, 2010. Date of current version March 29, 2010; date of current version June 03, 2010.

The authors are with the Laboratory of Electromagnetics and Acoustics (LEMA), Ecole Polytechnique Fédérale de Lausanne (EPFL), CH-1015 Lausanne, Switzerland (e-mail: athanasios.polymeridis@epfl.ch; juan.mosig@epfl. ch).

Digital Object Identifier 10.1109/TAP.2010.2046866
Since the very first applications of the mixed potential integral equation formulations, the weakly singular integrals over triangular domains appeared to be the most challenging of all, as the discretization into triangular elements is considered to be the most flexible one, being able to model surfaces of arbitrary shape. In general, weakly singular integrals are treated by using mainly the singularity subtraction method [4]-[9] or the singularity cancellation method [10]-[12]. Despite their widespread usage, both singularity subtraction and singularity cancellation methods fail to meet the requirements for an accurate and efficient numerical integration of weakly singular integrals.

On the other hand, some very promising new methods have appeared in the literature that seem to outperform the traditional techniques. In [13], for instance, a method originated in the context of mechanics, which utilizes a series of coordinate transformations followed by an appropriate Duffy transform [14] is presented. Similarly, a direct approach for the evaluation of hyper-singular static surface integrals has been introduced in [15]. Moreover, the later direct evaluation method was generalized by the first author for the case of the weakly singular integrals over coincident triangular elements [16]. The only drawback of these methods is their limited applicability together with their highly analytical complexity. Hence, it is quite difficult to generalize them in order to deal with different types of integrands and/or integration domains.

The present work is motivated mostly by our recent results presented in [16], where it is obvious that in both singularity subtraction and singularity cancellation methods the accuracy as well as the efficiency are limited by the remaining numerical integrations of smooth functions (without blow up singularities). Moreover, in some recent publications [11], [12], [17]-[19] it was clearly demonstrated that in many cases the near-singular potential integration (the inner 2-D integration) is more challenging than the singular one. Therefore, a reasonable resolution in the never ending quest for a machine precision general-purpose code, not only for 4-D weakly singular integrals but also for 4-D hyper-singular and general near-singular integrals, could be given by the combination of semi-analytical formulations together with appropriate cubature rules [20].

In this manuscript, we present generalized Cartesian product rules based on the double exponential quadrature formulas. These formulas are well known in the mathematical literature since their introduction in the mid-seventies by Takahasi and Mori [21]-[24]. In a recent paper, they have been compared with other numerical alternatives and hailed as one of the most promising high precision quadrature schemes [25]. However, 
they remain mostly unknown and unused by the computational electromagnetics community. In this paper, we first briefly recall for the sake of completeness and internal coherence the main steps of the technique, following the excellent summary given in [25]. Then, the double exponential formulas are integrated with classic methods for the computation of weakly singular and near-singular 2-D and 4-D integrals. The resulting overall scheme is compared with the most representative alternatives based on Gauss formulas, showing a significant improvement in almost all of the most challenging cases.

\section{The Double Exponential QuAdRATURe Rule}

Standard interpolatory quadrature rules like Newton-Cotes and Gauss formulas can normally be used for integrands that are regular at the endpoints of the integration interval. On the other hand, although Gauss-Jacobi formulas have been widely used for integrands with infinite derivatives or integrable singularities at the endpoints, the type of singularity that can be treated by such formulas is quite limited. Coming to fill this gap, the double exponential quadrature rule is not based on ad hoc transformations or very specific weight functions, but on an appropriate variable transformation which results in general purpose quadrature formulas so robust and efficient that deserve a prominent place in standard mathematical subroutine libraries.

As it is well known, the trapezoidal rule with an equal mesh size $h$ gives highly accurate results for analytic functions over $(-\infty, \infty)$. In fact, it was proved in [26] that for an integral of an analytic function over $(-\infty, \infty)$ the trapezoidal rule with an equal mesh size is asymptotically optimal among formulas with the same density of sampling points. The optimality of the trapezoidal formula turned out to play a crucial role in the process of the discovery by Takahasi and Mori [21] of the double exponential formula. We now briefly outline the main steps leading to the development of a practical implementation of the double exponential quadrature formula. Additional details can be found in [25].

\section{A. Double Exponential Transformation}

Without loss of generality, we will confine ourselves to the following integral over $(-1,1)$ :

$$
\mathrm{I} f=\int_{-1}^{1} f(x) d x
$$

Since Takahasi and Mori had already proved the optimality of the trapezoidal formula over $(-\infty, \infty)$, it was quite natural that they focused on a variable transformation which maps the original interval of integration onto $(-\infty, \infty)$ [21], i.e.,

$$
x=\phi(t)
$$

which we assume is analytic over $-\infty<t<\infty$ and satisfies

$$
\phi(-\infty)=-1, \quad \phi(+\infty)=1
$$

Hence, the original integral is given by

$$
\mathrm{I} f=\int_{-\infty}^{\infty} f(\phi(t)) \phi^{\prime}(t) d t
$$

which is solved with the help of the trapezoidal formula

$$
\mathrm{I}_{h} f=h \sum_{k=-\infty}^{\infty} f(\phi(k h)) \phi^{\prime}(k h) .
$$

The remaining point is now the optimal selection of the change of variables $x=\phi(t)$. The main idea, based on the Euler-Maclaurin formula [27], is to select a transformation $\phi(t)$ such as all its derivatives tend exponentially to zero for large values (positive and negative) of $t$. Then, even if $f(x)$ or its derivatives have an integrable algebraic singularity at one or both endpoints, they will disappear within the smooth and fast convergence of the new integrand $f(\phi(t)) \phi^{\prime}(t)$ at infinity. In these cases, according to the Euler-Maclaurin argument, the quadrature error should decrease faster than any power of $h$.

As described in [25], based on the above reasoning, Takahasi and Mori came out with a very interesting variable transformation

$$
\begin{aligned}
x & =\phi(t)=\tan h\left(\frac{\pi}{2} \sin h(t)\right) \\
\phi^{\prime}(t) & =\frac{\frac{\pi}{2} \cos h(t)}{\cos h^{2}\left(\frac{\pi}{2} \sin h(t)\right)} \approx O\left(\exp \left(-\frac{\pi}{2} \exp (|t|)\right)\right)
\end{aligned}
$$

as $|t| \rightarrow \infty$. Due to this double exponential decay, (6) is called the double exponential (DE) transformation. An alternate frequently used name, based again on (6), is the "tanh-sinh" technique. If we truncate (5) at $k= \pm n$, we get the $\mathrm{DE}$ formula,

$\mathrm{I}_{h}^{(N)} f=h \sum_{k=-n}^{n} f\left(\tanh \left(\frac{\pi}{2} \sinh (k h)\right)\right) \frac{\frac{\pi}{2} \cosh (k h)}{\cosh ^{2}\left(\frac{\pi}{2} \sinh (k h)\right)}$

where $N=2 n+1$ is the number of the quadrature rule's abscissas. The key feature of the DE formula's superior performance compared to other quadrature rules designed to handle endpoint singularities lies in the fact that via the DE transformation one can approach the endpoint singularity as close as one wants, because the DE rule has an infinite number of points in the neighborhood of the endpoints [22]. In cases where the integrand has a blow up singularity at an endpoint, this scheme permits one to sum terms with abscissas very close to the endpoints until the rapidly decreasing weights overwhelm the large function values [25].

\section{B. On the Implementation of the Double Exponential Quadrature Rule}

Although it is very easy to compute the weights and abscissas of the DE quadrature rule, we encounter two significant problems in the actual coding process. According to [24], the problems arising from the careless coding may be one of the reasons that prevented the spread of the DE formula. More specifically, 
TABLE I

Number of INTEgRATION PoINTS $(N)$ FOR THE DE RUlE IN TERMS OF THE ASSOCIATED LEVEL OF THE QUADRATURE RULE $(M)$

\begin{tabular}{c|cccccccc}
\hline$M$ & 0 & 1 & 2 & 3 & 4 & 5 & 6 & 7 \\
\hline$N$ & 7 & 13 & 25 & 51 & 101 & 203 & 405 & 809 \\
\hline
\end{tabular}

the first and most severe problem is the loss of significant digits: If $f(x)$ has a singularity at the endpoint like $(1 \pm x)^{-1+\mu}$, where $\mu$ is a small positive constant, we often encounter a large error due to the loss of significant digits for $x$ very close to \pm 1 . One way to overcome the aforementioned problem is to compute and store the values $\Xi=1+x$ and $\mathrm{H}=1-x$ in addition to the actual weights and abscissas. Of course, this modification requires the a priori identification of those binomials in the integrand, reducing the overall generality of the DE scheme.

The second problem is the numerical underflow and overflow which arises in the denominator of the weights in (8). More specifically, the constraints that are imposed due to the double precision format lead, after some algebraic manipulation, to the following (safe) choice in the construction of the DE quadrature rule:

$$
\max (|t|)=n h=6 .
$$

\section{Final Double Exponential Formulas}

Taking into account the aforementioned constraints we proceed to the construction of the DE formula, according to the following parametrization, which is slightly different from the one adopted in the numerical experiments presented in [25] (aiming at 400 significant digits)

$$
\begin{aligned}
& n=6 \cdot 2^{M}, \\
& h=1 / 2^{M}
\end{aligned}
$$

where $M$ is the so-called level of the quadrature rule. This choice fulfils the constraint (9) to avoid numerical underflow and overflow. Besides the parametrization, one could follow different strategies in the final algorithms. The most suitable candidate for a general purpose integration scheme, though, is based on truncating the series of weights and abscissas at the point $x_{k}, k>0$ for which the following inequality holds: $1-x_{k}<$ eps, where eps stands for the machine precision in double precision arithmetic, ensuring that $1-x$ and $1+x$ are never equal to zero. The final truncated number of points is equal to $N=2 k+1$, as shown in Table I, for each level of the rule.

Another possible variant could be derived without the aforementioned truncation together with the pre computation of $\Xi$ and $\mathrm{H}$. In that case, the number of integration points is given by $N=2 n+1=2\left(6 \cdot 2^{M}\right)+1$, almost double compared to our preferred choice. This formula guaranties much higher accuracy in some specific problems, but its problem oriented nature is limiting dramatically the repertoire of possible applications and, hence, will be excluded from our study.

\section{Extension to Generalized Cartesian Product Rules}

As described above, DE quadrature rule does not integrate exactly any polynomial and therefore it doesn't belong to the family of the interpolatory quadrature formulas like NewtonCotes, Gauss, Radau, Lobatto and many others. Nevertheless, it turns out to be highly accurate for the integration of analytic functions with algebraic or/and logarithmic singularities at the endpoints of the integration interval, as will be shown in the sample test integrals following in the next section. This is exactly the reason that engages us in the quest for the construction of multidimensional integration schemes based on sophisticated quadrature formulas like DE rule, which are suitable for the treatment of functions with boundary weakly singular behavior.

A first attempt for the generalization of the DE quadrature rule in 2-D and 3-D integration formulas was given in [28]. More specifically, a progressive strategy was utilized where the integration interval is divided into subdomains and, after a specific search pattern, the optimal abscissas and weights are found. The main drawback, though, of this method is its problem oriented nature, since the progressive search has to be performed for each new integrand.

On the other hand, another research group followed a different approach while trying to find optimal cubatures for weakly singular, strongly (or Cauchy) singular and hyper-singular multidimensional integrals [29], [30]. In [29], a combination of Gauss product rules and DE formulas for the integration of 2-D singular integrals, arising in the context of computational mechanics, was implemented. Later, the same team in [30] focused on various 4-D singular integrals. The 4-D integrals are treated in the following way: First, an analytical integration is performed for the inner 2-D integral. Then, the type of singularity of the aforementioned analytical results as a function of the outer variables of integration is studied, in order to give some indications about the integration formulas needed for the remaining outer 2-D integral. In the end, again, generalized 2-D Cartesian product rules for the integration of at most boundary weakly singular functions are proposed based on a combination of 1-D Gauss product rules and DE quadrature rule.

As a general comment, we could add that a hybrid scheme with analytical 2-D inner integrations and numerical 2-D outer integrations, as in [30], clearly reduces the computation cost and increases the accuracy. Furthermore, the study of the analytical results as function of the outer variables helps significantly in the suitable choice of the basic quadrature rules used for the construction of optimal multidimensional integration formulas. Of course, DE quadrature rule stands as one of the most appealing choices due to its unmatched precision in integrating functions with endpoint algebraic or/and logarithmic singularities.

In the computational electromagnetics community there are generally two main families of methods for the solution of weakly singular integrals arising in the mixed potential integral equation formulations. Starting with the singularity subtraction method, it is well known that the integrand of the final 4-D integral may be non-singular, but with infinite derivatives. Also, the subtracted function can only be evaluated for specific 
geometries, leading to limited applicability. On the other hand, in the singularity cancellation method only the computation of the potential (inner) integrals to machine precision has been studied, and most of the authors imply that this is enough in order to assure highly accurate results for the original 4-D integrals. Unfortunately, this is not the case, since a simple study reveals that the integrand of the outer integral may still have infinite derivatives at the endpoints of the integration interval. In this manuscript, following the basic philosophy in [30], we utilize a DE rule as the main building block in the generalized Cartesian product rules for the solution of the aforementioned shortcomings.

\section{NUMERICAL RESULTS}

In this section, various numerical results will be presented in order to illustrate the worthiness of incorporating the DE quadrature rule in the construction of generalized Cartesian product rules for the solution of multidimensional weakly singular integrals arising in the mixed potential integral equation formulations. After some test 1-D and 2-D problems, we will show the results of some representative numerical experiments including different approaches, like singularity cancellation and singularity subtraction methods.

\section{A. Sample 1-D and 2-D Test Integrals}

In the beginning, it would be intuitive to give some results for a selection of 1-D test integrals found in [25]. The importance of such numerical experiments is twofold: first we validate the $\mathrm{DE}$ quadrature rule, as proposed in Section II, and, second, we observe its limitations for various types of endpoint singularities. The selected groups of test integrals (keeping the same numbering as in [25]) are listed below:

- 5: continuous function on finite interval, but with an infinite derivative at an endpoint;

- 7, 8, 10: functions on finite intervals with an integrable singularity at an endpoint.

$$
\begin{array}{llll}
\text { 5: } & \int_{0}^{1} \sqrt{t} \ln (t) d t, & 7: & \int_{0}^{1} \frac{\sqrt{t}}{\sqrt{1-t^{2}}} d t \\
\text { 8: } & \int_{0}^{1} \ln ^{2}(t) d t, \quad 10: & \int_{0}^{\pi / 2} \sqrt{\tan (t)} d t .
\end{array}
$$

A straightforward application of the DE formulas as well as the Gauss-Legendre formulas leads to the relative error presented in Fig. 1, in terms of the associated level $M$ of the quadrature rule. For a fair comparison, we keep obviously the same number of integration points in both Gauss and DE formulas. Clearly, for the case of the test integral \#5, function with infinite derivative at an endpoint, the DE formulas converge to the exact solution, while Gauss based formulas give very poor results. The same behavior is also observed for the test integral \#8. For the other two test integrals \#7 and \#10, functions with an integrable singularity at an endpoint, the DE formulas cannot converge to the exact solution, but in any case their performance is by far superior to the performance of the Gauss rules.

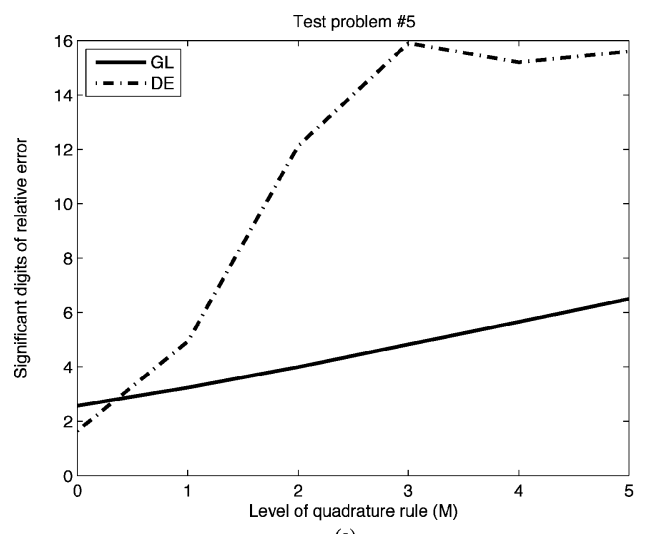

(a)

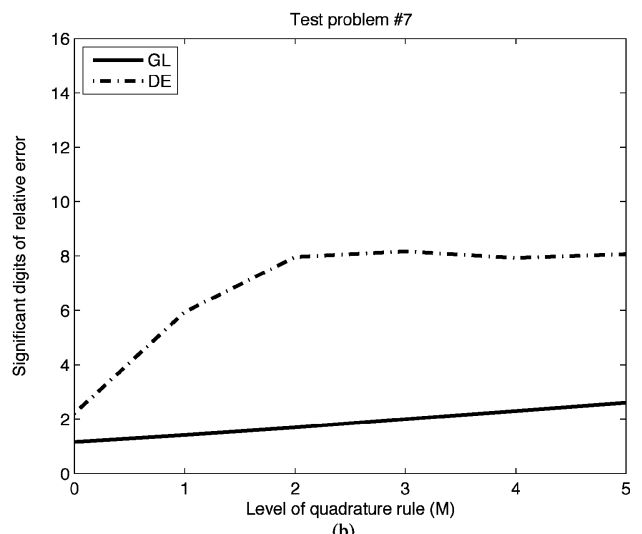

Test problem \#8
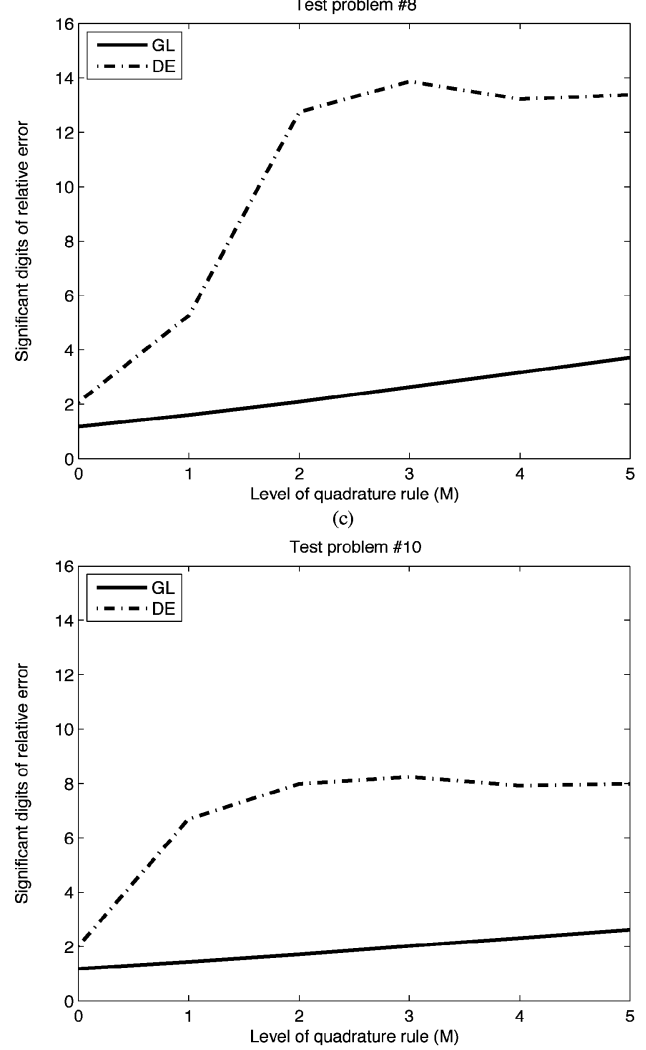

(d)

Fig. 1. Relative error in calculating the test integrals in (11). (a) Test integral \#5. (b) Test integral \#7. (c) Test integral \#8. (d) Test integral \#10. 


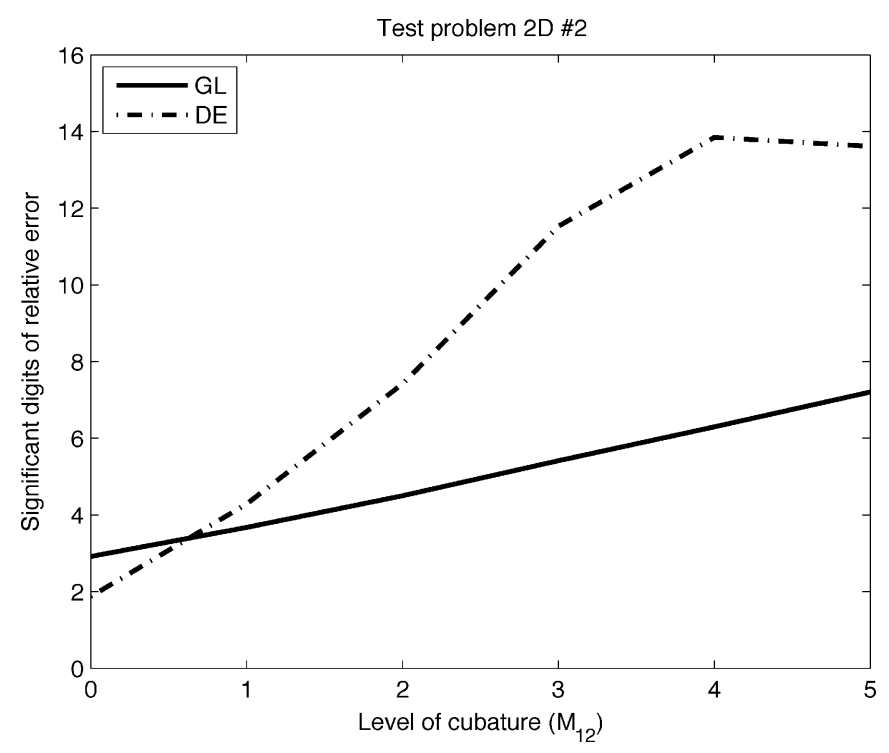

Fig. 2. Relative error in calculating the test integral (12).

Finally, we choose a 2-D test integral \#2 with endpoint singularities $(x=y= \pm 1)$ found in [28]:

$$
\begin{aligned}
I_{2}^{2 \mathrm{D}} & =\int_{-1}^{1} \int_{-1}^{1} \frac{1}{\sqrt{1-x^{2} y^{2}}} d x d y \\
& \approx \sum_{i}^{M_{1}} \sum_{j}^{M_{2}} c_{i} c_{j} f\left(x_{i}, y_{j}\right)
\end{aligned}
$$

where $M_{1}=M_{2}=M_{12}$ is the level of the 1-D quadrature rules ( $M_{12}$ is also called level of the cubature) used in the construction of the 2-D Cartesian product rule. Hence, each cubature utilizes the same quadrature rule for each of the dimensions. As shown clearly in Fig. 2, again, DE based cubatures succeed in giving highly accurate results compared to the Gauss based cubatures. To account for the incidental presence of error propagation effects, we cautiously assume a result to be numerically exact if its relative error is lower than $10^{-13}$, as suggested in [12].

\section{B. Weakly Singular Potential Integrals}

Moving to some more challenging problems, we choose the weakly singular potential integral

$$
I_{s}^{\text {potential }}=\int_{E_{Q}} \frac{e^{-j k R}}{R} d A_{Q}
$$

where $E_{Q}$ is the unit triangle defined by the following vertices: $\mathbf{r}_{1}=(0,0,0), \mathbf{r}_{2}=(1,0,0), \mathbf{r}_{3}=(0,1,0), R$ is the distance function and $k=1$. The observation point is given by $\mathbf{r}=(0.1,0.1,0)$. The singularity lies in the integration interval, for which case is very hard to find efficient cubatures. Hence, we split the integral into three subintegrals, isolating the singularity only at one vertex of the new triangles. Next, we proceed to the fully numerical integration of all three 2-D integrals via generalized Cartesian product rules based on Gauss-Legendre and DE formulas.
The reference solution of the integral (absolute error less than $\left.10^{-14}\right)$ is directly copied from [12],

$$
I_{\text {ref }}=1.89857266176847-j 0.309643085636859 .
$$

In Fig. 3 the relative error in calculating the real part (singular portion) and the imaginary part (nonsingular portion) of the weakly singular potential integral (13) using the DE quadrature rule as well as basic Gauss formulas in 2-D generalized Cartesian product rules, is shown. The level of cubature $M_{12}$ is equal to the level of the 1-D quadrature rules $M_{1}, M_{2}$, i.e., $M_{12}=M_{1}=M_{2}$. By a simple inspection of the figures, it is easy to come to the conclusion that DE cubatures converge to the numerically exact solution for the most challenging case (the singular real part). As it could be expected, there is no interest in using DE rules for non-singular functions (the imaginary part) where Gauss-Legendre is optimally suited.

\section{4-D Weakly Singular Integrals Over Triangles via Singularity Cancellation}

Next, we deal with the far more interesting 4-D weakly singular integral arising in the mixed potential integral equation formulations solved via a Galerkin triangular discretization together with the linear Rao-Wilton-Glisson basis functions. More specifically, the contribution due to the scalar potential is given by

$$
I_{s}=\int_{E_{P}} \int_{E_{Q}} \frac{e^{-j k R}}{R} d A_{Q} d A_{P}
$$

where $E_{P} \equiv E_{Q}$ is the unit triangle defined by the following vertices: $\mathbf{r}_{\mathbf{1}}=(0,0,0), \mathbf{r}_{\mathbf{2}}=(1,0,0), \mathbf{r}_{3}=(0,1,0)$ and $k=1$. Following the basic idea of the singularity cancellation method, we get

$$
I_{s}=\int_{E_{P}} I_{Q}^{\text {potential }} d A_{P}
$$

where the outer integration over the triangle $E_{P}$ is numerically computed using a generalized Cartesian product rule. On the other hand, the inner or potential integral is reduced to a 1-D smooth integral via an appropriate coordinate transformation and a further analytical evaluation. The remaining 1-D inner integral is computed via a simple quadrature rule.

The reference solution of the integral (absolute error less than $10^{-16}$ ) is directly copied from [13],

$$
I_{\mathrm{ref}}=0.952716973790348-j 0.240945897671652 .
$$

In Figs. 4 and 5 the relative error in calculating the real part (singular portion) of the 4-D weakly singular integral (15) using the singularity cancellation method together with a generalized Cartesian quadrature rule based on the DE quadrature rule and the Gauss-Legendre formulas, is shown. More specifically, for the results in Fig. 4 we used a standard Gauss-Legendre quadrature rule for the remaining 1-D integral, while for the results in 


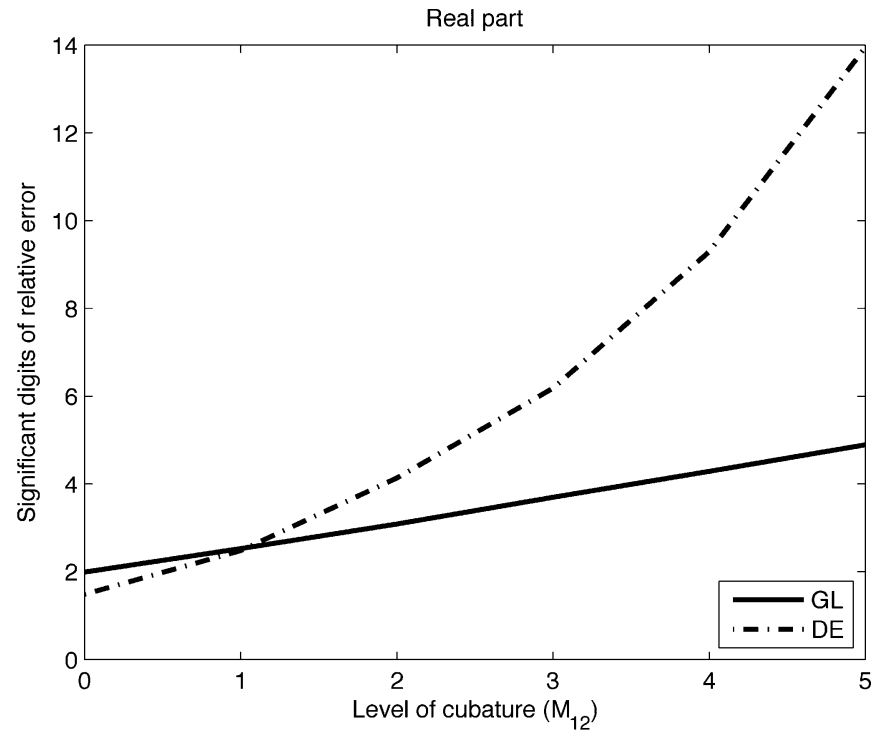

(a)

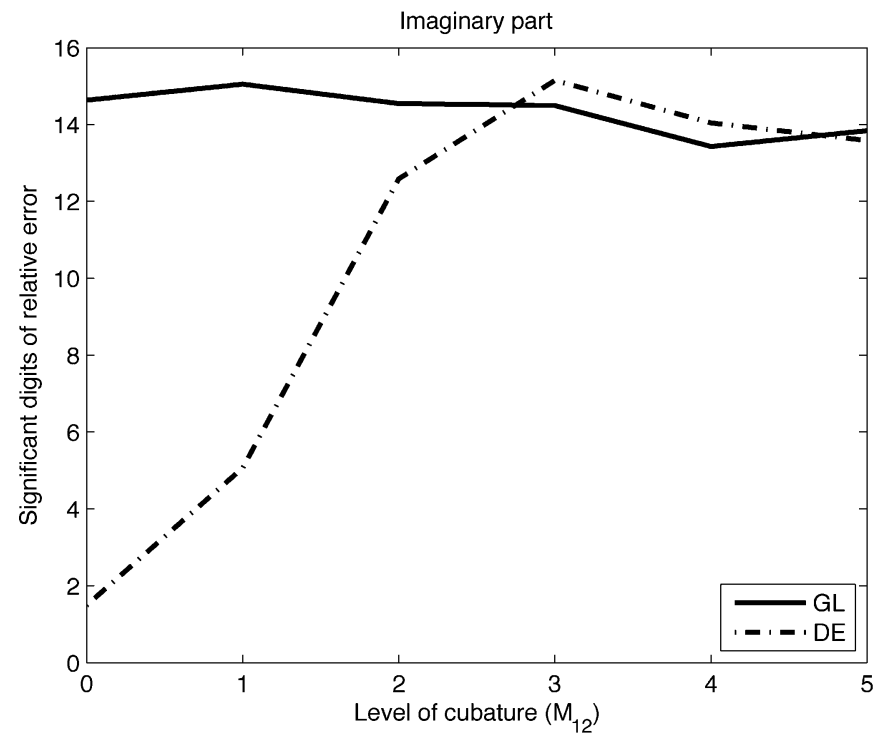

(b)

Fig. 3. Relative error in calculating the weakly singular potential integral

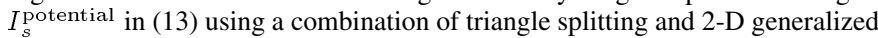
Cartesian product rules based on Gauss-Legendre and DE quadrature rules. (a) Real part. (b) Imaginary part.

Fig. 5 we utilized the DE quadrature rule, again for the inner 1-D integration. The level of the outer cubature $M_{23}$ is equal to the level of each quadrature rule employed in the construction of the 2-D formulas, i.e., $M_{23}=M_{2}=M_{3}$. A simple comparison of the aforementioned figures leads to the safe conclusion that only the 3-D generalized Cartesian product rule which is solely based on $\mathrm{DE}$ quadrature rules $\left(\mathrm{DE}^{2}-\mathrm{DE}\right)$ can give numerically exact results, as is clearly depicted in Fig. 5(b).

The performance of the scheme that is presented in this manuscript is of paramount importance, since it is the first time that the singularity cancellation method can produce results close to the machine precision with a reasonable number of integration points. A major factor that affects crucially the accuracy of the

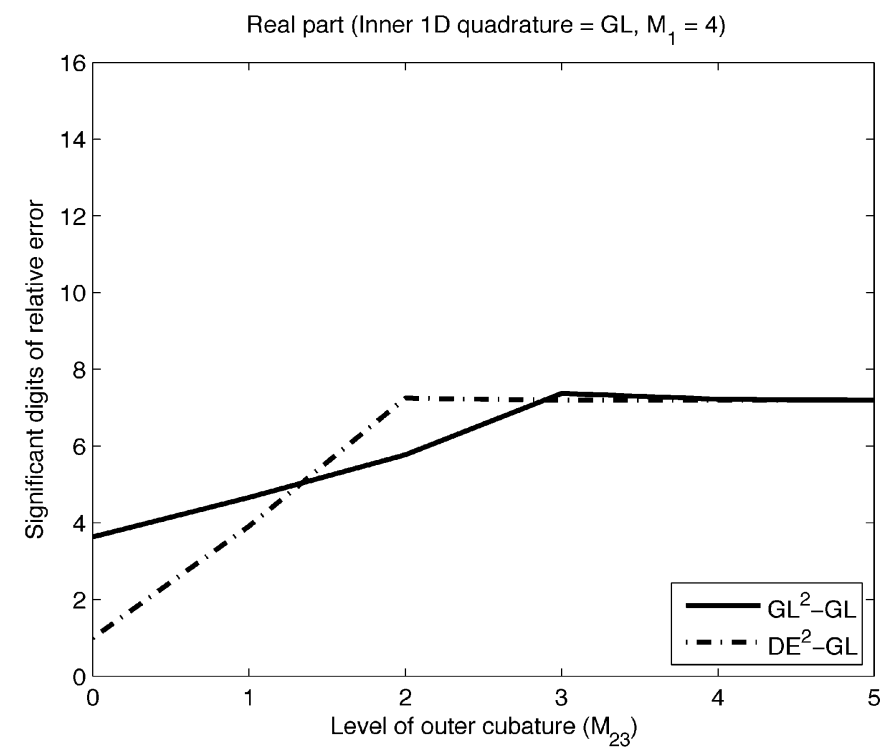

(a)

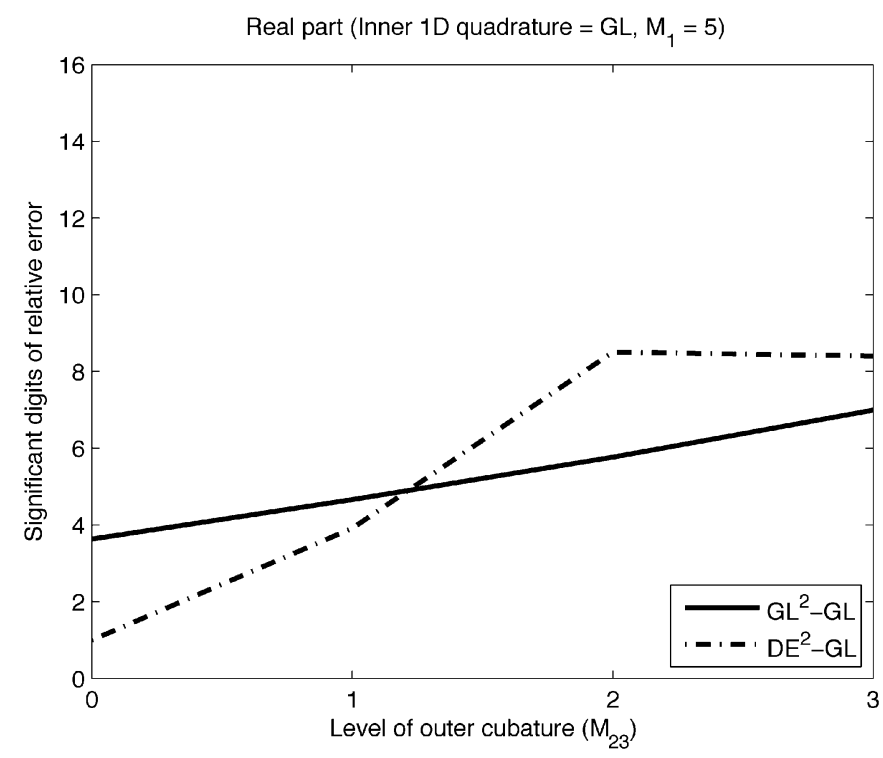

(b)

Fig. 4. Relative error in calculating the real part of the 4-D weakly singular integral $I_{s}$ in (15) via singularity cancellation and 3-D generalized Cartesian product rules based on Gauss-Legendre and DE quadrature rules. For the inner 1-D integral a Gauss-Legendre rule is utilized. (a) Level of inner integration rule: $M_{1}=4$. (b) Level of inner integration rule: $M_{1}=5$.

3-D numerical integration seems to be the behavior of the inner integral as a function of outer's integration variables. Generally, the outer integration hasn't been thoroughly studied in previous publications, where it was believed that machine precision results of the inner integral would physically lead to highly accurate results also for the final 4-D weakly singular integral by a straightforward implementation of Gaussian cubatures. One could easily jump to the conclusion that although the singularity is canceled, numerical shortcomings are encountered in the integration of the remaining function due to the non smooth behavior of its higher order derivatives. 


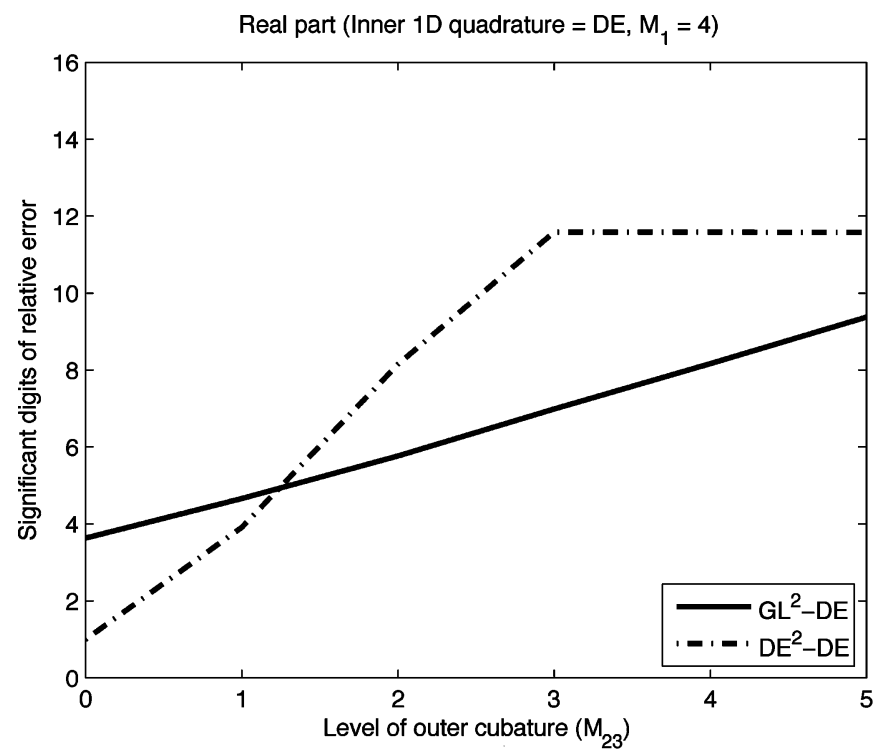

(a)

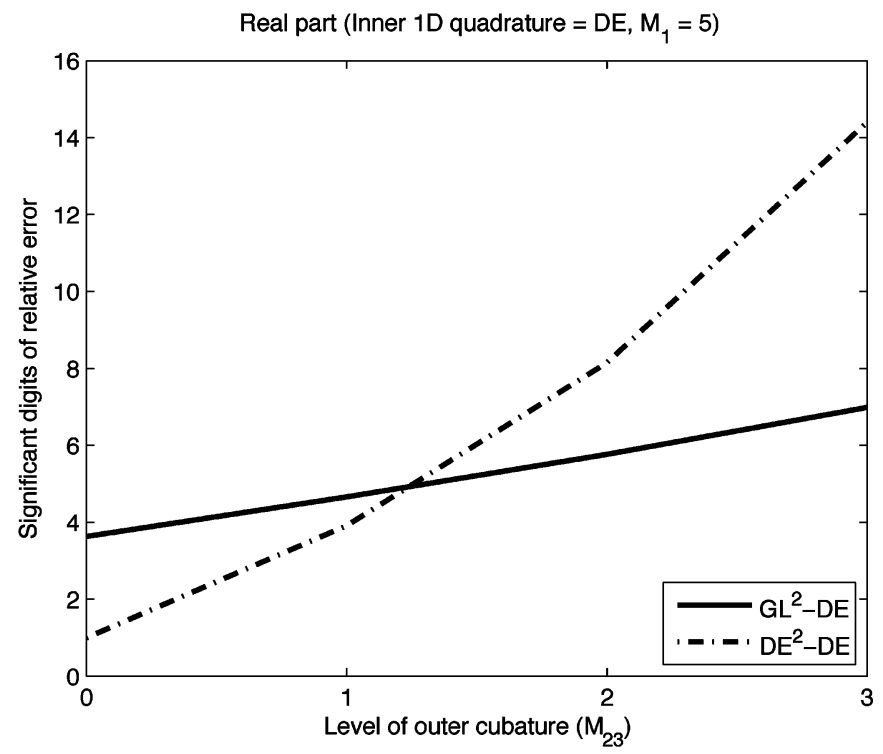

(b)

Fig. 5. Relative error in calculating the real part of the 4-D weakly singular integral $I_{s}$ in (15) via singularity cancellation and 3-D generalized Cartesian product rules based on Gauss-Legendre and DE quadrature rules. For the inner 1-D integral a DE rule is utilized. (a) Level of inner integration rule: $M_{1}=4$. (b) Level of inner integration rule: $M_{1}=5$.

\section{4-D Weakly Singular Integrals Over Quadrangles via Semi-Analytical Method}

As a final test, we shall present sample numerical results in the context of the 4-D weakly singular integrals over quadrangular domains for the static kernel. More specifically, the 4-D integral to be solved is written as follows:

$$
I_{\text {static }}=\int_{E_{P}} \int_{E_{Q}} \frac{1}{R} d A_{Q} d A_{P}
$$

where $E_{P}$ and $E_{Q}$ are general quadrilaterals and $R$ is the distance function. The most common approach for treating the aforementioned integral is the so-called semi-analytical method.
The key feature of this method is the analytical evaluation of the potential (inner) integral,

$$
I_{\text {static }}^{\text {potential }}=\int_{E_{Q}} \frac{1}{R} d A_{Q}
$$

as explained in [4] and [5]. The second and final step is the numerical computation of the remaining 2-D integral,

$$
I_{\text {static }}=\int_{E_{P}} I_{\text {static }}^{\text {potential }} d A_{P}
$$

Without loss of generality, we confine ourselves to the case of square domains (with sides equal to 2). Fig. 6 depicts the relative error in calculating the 4-D weakly singular integral (18) utilizing the analytical expressions provided in [4] together with a 2-D generalized Cartesian product rule based on the $\mathrm{DE}$ quadrature rule and the Gauss-Legendre formulas. More specifically, in Fig. 6(a), (b) and (c)-(d), we examine respectively the self-term case (coinciding squares), the orthogonal case (orthogonal squares sharing one edge) and the parallel case (parallel squares separated by distance $d$ ). The reference results are derived with the help of the complete analytical formulas provided in [9].

As with the previous examples, generalized Cartesian product rules based on the DE formula outperform the standard GaussLegendre cubatures for the solution of the most challenging cases, i.e., the self-term case (Fig. 6(a)) and the parallel case when the distance between the elements is very small (Fig. 6(c)). Obviously, for non-singular cases Gauss-Legendre still remains the optimal solution. Trying to give a fair explanation for the poor results of the Gauss-Legendre cubatures or the superior performance of the proposed DE based schemes, we analyze the behavior of the analytically evaluated results of the inner 2-D integral in terms of the observation point, i.e., the point given by the outer cubature in the final computation of the 4-D integral.

For example, the potential integral for the observation points $\mathbf{r}_{\mathbf{o}_{1}}=(-1,0, c)$ and $\mathbf{r}_{\mathbf{o}_{2}}=(c, 0,0)$, where $c=-2: 2$, due to the source square with the following vertices: $\mathbf{r}_{1}=(-1,-1,0)$, $\mathbf{r}_{2}=(1,-1,0), \mathbf{r}_{3}=(1,1,0)$ and $\mathbf{r}_{4}=(-1,1,0)$, is presented in Fig. 7. Clearly, in the second case (encountered in the evaluation of the self-term), the potential has infinite derivatives as the observation point passes from one edge of the source square. The aforementioned behaviors, together with the overall discussion in previous sections, come to elucidate the actual causes for the performance of the integration schemes used in our numerical experiments.

\section{CONCLUSION}

Novel generalized Cartesian product rules are presented for the computation of various multidimensional weakly singular integrals, arising in mixed potential integral equation formulations. The proposed formulas utilize the double exponential quadrature rule, ideally suited for the integration of functions with endpoint singularities. Due to the use of such non interpolatory quadrature rules, the algorithms presented in this manuscript can lead, together with common singularity subtraction 


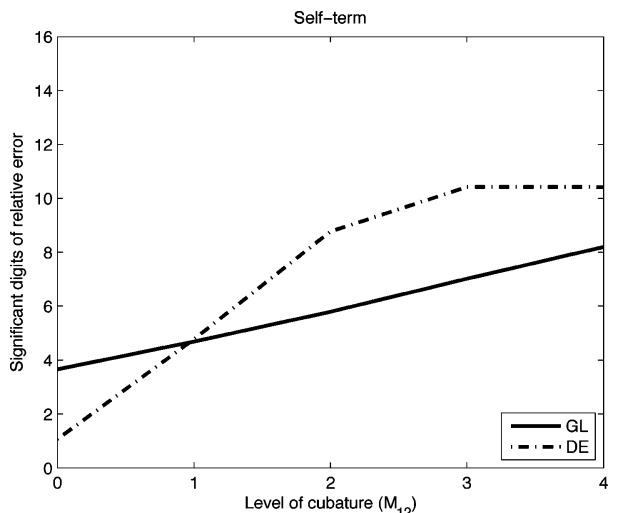

(a)

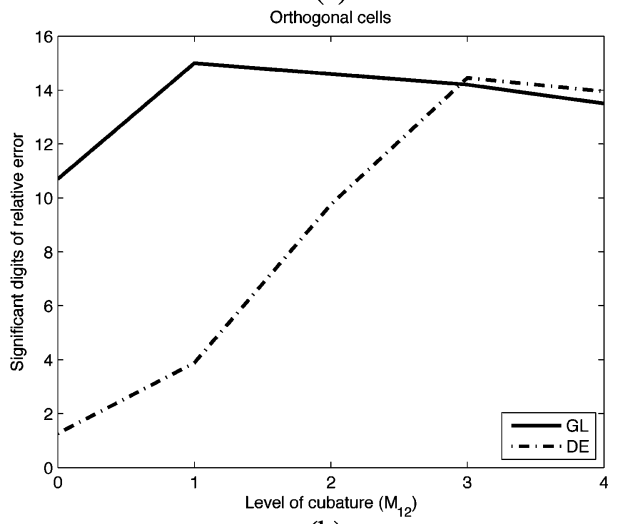

(b)

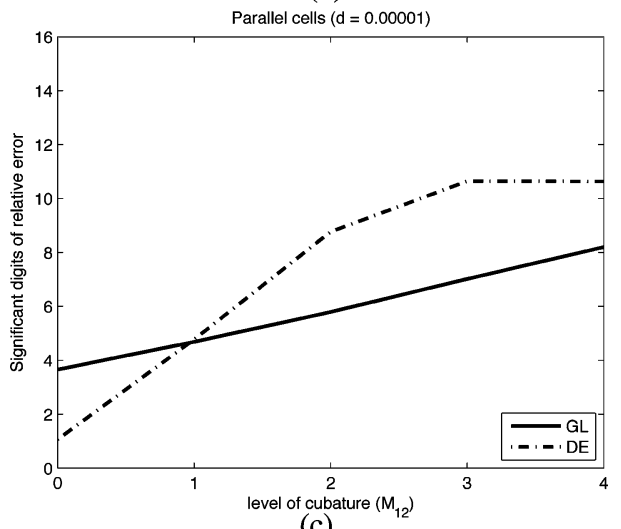

(c)

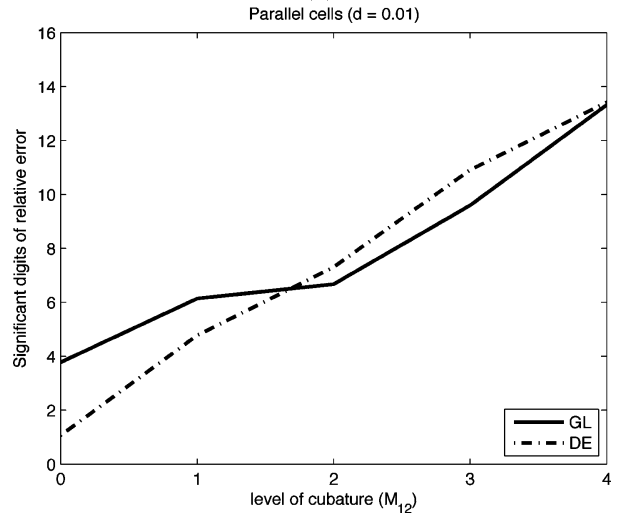

(d)

Fig. 6. Relative error in calculating the 4-D weakly singular integral (18) (with $k=1$ ) utilizing the analytical expressions provided in [4] together with a 2-D generalized Cartesian product rule based on DE and Gauss-Legendre quadrature rules. (a) Self-term. (b) Orthogonal cells. (c) Parallel cells $(d=0.00001)$. (d) Parallel cells $(d=0.01)$.

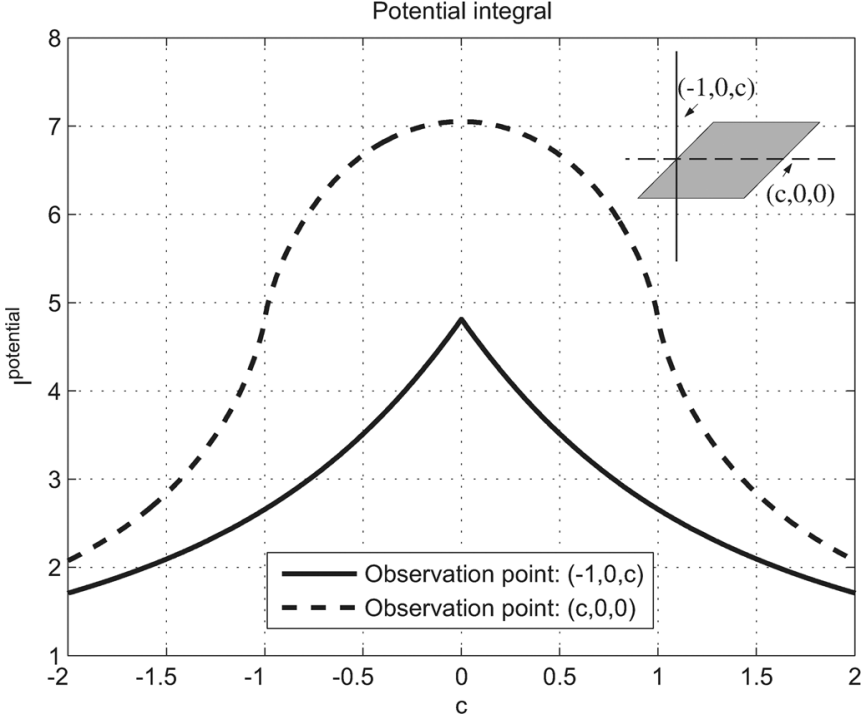

Fig. 7. Behavior of the analytically derived potential integral (20) for a square with the following vertices: $\mathbf{r}_{1}=(-1,-1,0), \mathbf{r}_{2}=(1,-1,0)$, $\mathbf{r}_{3}=(1,1,0)$ and $\mathbf{r}_{4}=(-1,1,0)$ and different observation points $\mathbf{r}_{\mathbf{o}_{1}}=(-1,0, c)$ and $\mathbf{r}_{\mathbf{o}_{2}}=(c, 0,0)$, respectively.

and cancellation techniques, to unmatched accuracy. The superior performance of the proposed method is verified in comparison with standard interpolatory cubatures through a series of representative numerical experiments. Moreover, in many cases the accuracy of the integration via double exponential based formulas is close to the machine precision, working on typical double precision arithmetic. Finally, the detailed analysis presented herein forms the backbone for the treatment of a plethora of cumbersome integrals, like strongly singular and hyper-singular integrals arising in various integral equation formulations, always in the context of computational electromagnetics.

\section{REFERENCES}

[1] S. M. Rao, D. R. Wilton, and A. W. Glisson, "Electromagnetic scattering by surfaces of arbitrary shape," IEEE Trans. Antennas Propag., vol. 30, no. 5, pp. 409-418, May 1982.

[2] R. F. Harrington, Field Computation by Moment Methods. New York: Macmillan, 1983

[3] J. R. Mosig, R. C. Hall, and F. E. Gardiol, "Numerical analysis of Microstrip patch antennas," in Handbook of Microstrip Antennas, James and Hall, Eds. London: IEE-Peter Peregrinus, 1989.

[4] D. R. Wilton, S. M. Rao, A. W. Glisson, D. H. Schaubert, O. M. AL-Bundak, and C. M. Butler, "Potential integrals for uniform and linear source distributions on polygonal and polyhedral domains," IEEE Trans. Antennas Propag., vol. 32, no. 3, pp. 276-281, Mar. 1984.

[5] R. D. Graglia, "On the numerical integration of the linear shape functions times the 3-D Green's function or its gradient on a plane triangle," IEEE Trans. Antennas Propag., vol. 41, no. 10, pp. 1448-1455, Oct. 1993.

[6] T. F. Eibcrt and V. Hansen, "On the calculation of potential integrals for linear source distributions on triangular domains," IEEE Trans. Antennas Propag., vol. 43, no. 12, pp. 1499-1502, Dec. 1995.

[7] P. Arcioni, M. Bressan, and L. Perregrini, "On the evaluation of the double surface integrals arising in the application of the boundary integral method to 3-D problems," IEEE Trans. Microw. Theory Tech., vol. 45, no. 3, pp. 436-439, Mar. 1997.

[8] S. Järvenpää, M. Taskinen, and P. Ylä-Oijala, "Singularity subtraction technique for high-order polynomial vector basis functions on planar triangles," IEEE Trans. Antennas Propag., vol. 54, no. 1, pp. 42-49, Jan. 2006. 
[9] S. López-Peña and J. R. Mosig, "Analytical evaluation of the quadruple static potential integrals on rectangular domains to solve 3-D electromagnetic problems," IEEE Trans. Magn., vol. 54, no. 3, pp. 1320-1323, Mar. 2009.

[10] L. Rossi and P. J. Cullen, "On the fully numerical evaluation of the linear-shape function times the 3-D Greens function on a plane triangle," IEEE Trans. Microw. Theory Tech., vol. 47, no. 4, pp. 398-402, Apr. 1999.

[11] M. A. Khayat and D. R. Wilton, "Numerical evaluation of singular and near-singular potential integrals," IEEE Trans. Antennas Propag., vol. 53, no. 10, pp. 3180-3190, Oct. 2005.

[12] R. D. Graglia and G. Lombardi, "Machine precision evaluation of singular and nearly singular potential integrals by use of Gauss quadrature formulas for rational functions," IEEE Trans. Antennas Propag., vol. 56, no. 4, pp. 981-998, Apr. 2008.

[13] D. J. Taylor, "Accurate and efficient numerical integration of weakly singular integrals in Galerkin EFIE solutions," IEEE Trans. Antennas Propag., vol. 51, no. 7, pp. 1630-1637, July 2003.

[14] M. G. Duffy, "Quadrature over a pyramid or cube of integrands with a singularity at a vertex," SIAM, J. Numer. Anal., vol. 19, no. 6, pp. $1260-1262,1982$.

[15] L. J. Gray, A. Salvadori, A. V. Phan, and A. Mantic, "Direct evaluation of hypersingular Galerkin surface integrals. II," Electronic Journal of Boundary Elements, vol. 4, no. 3, pp. 105-130, 2006.

[16] A. G. Polimeridis and T. V. Yioultsis, "On the direct evaluation of weakly singular integrals in Galerkin mixed potential integral equation formulations," IEEE Trans. Antennas Propag., vol. 56, no. 9, pp. 3011-3019, Sep. 2008.

[17] Ismatullah and T. F. Eibert, "Adaptive singularity cancellation for efficient treatment of near-singular and near-hypersingular integrals in surface integral equation formulations," IEEE Trans. Antennas Propag., vol. 56, no. I, pp. 274-278, Jan. 2008.

[18] M. A. Khayat, D. R. Wilton, and P. W. Fink, "An improved transformation and optimized sampling scheme for the numerical evaluation of singular and near-singular potentials," IEEE Antennas Wireless Propag. Lett., vol. 7, pp. 377-380, 2008.

[19] P. W. Fink, D. R. Wilton, and M. A. Khayat, "Simple and efficient numerical evaluation of near-hypersingular integrals," IEEE Antennas Wireless Propag. Lett., vol. 7, pp. 469-472, 2008.

[20] Z. Wang, J. Volakis, K. Saitou, and K. Kurabayashi, "Comparison of semi-analytical formulations and Gaussian-quadrature rules for quasi-static double-surface potential integrals," IEEE Antennas Propag. Mag., vol. 45, no. 6, pp. 96-102, Dec. 2003.

[21] H. Takahasi and M. Mori, "Double exponential formulas for numerical integration," Publ. RIMS, Kyoto Univ., no. 9, pp. 721-741, 1974.

[22] M. Mori, "Quadrature formulas obtained by variable transformation and the DE-rule," J. Comput. Appl. Math., no. 112, pp. 119-130, 1985.

[23] M. Mori and M. Sugihara, "The double exponential transformation in numerical analysis," J. Comput. Appl. Math., no. 127, pp. 287-296, 2001.

[24] M. Mori, "The discovery of the double exponential transformation and its developments," Publ. RIMS, Kyoto Univ., no. 41, pp. 897-935, 2005.

[25] D. H. Bailey, K. Jeyabalan, and X. S. Li, "A comparison of three highprecision quadrature schemes," Exp. Math., vol. 3, no. 14, pp. 317-329, 2005.
[26] H. Takahasi and M. Mori, "Error estimation in the numerical integration of analytic functions," Rep. Comput. Centre Univ. Tokyo, no. 3, pp. 41-108, 1970

[27] A. R. Krommer and C. W. Ueberhuber, Computational integration. Philadelphia, PA: SIAM, 1998.

[28] V. U. Aihie and G. A. Evans, "A comparison of the error function and the tanh transformation as progressive rules for double and triple singular integrals," J. Comput. Appl. Math., no. 30, pp. 145-154, 1990.

[29] A. Aimi, M. Diligenti, and G. Monegato, "Numerical integration schemes for the BEM solution of hypersingular integral equations," Int. J. Numer. Methods Eng., vol. 45, pp. 1807-1830, 1999.

[30] A. Aimi and M. Diligenti, "Hypersingular kernel integration in 3D Galerkin boundary element method," J. Comput. Appl. Math., no. 138, pp. $51-72,2002$.

Athanasios G. Polimeridis was born in Thessaloniki, Hellas, in 1980. He received the Diploma degree in electrical engineering and the Ph.D. degree from the Department of Electrical and Computer Engineering, Aristotle University of Thessaloniki (AUTh), Thessaloniki, Hellas, in 2003 and 2008, respectively.

Since October 2008, he has been a Postdoctoral Research Fellow with the Laboratory of Electromagnetics and Acoustics, Ecole Polytechnique Fédérale de Lausanne (EPFL), Lausanne, Switzerland. His research interests include computational electromagnetics, with emphasis on the development and implementation of integral-equation based algorithms.

Juan R. Mosig (S'76-M'87-SM'94-F'99) was born in Cádiz, Spain. He received the Electrical Engineer degree from the Universidad Politécnica de Madrid, Madrid, Spain, in 1973, and the Ph.D. degree from the Ecole Polytechnique Fédérale de Lausanne (EPFL), Lausanne, Switzerland, in 1983.

In 1976, he joined the Laboratory of Electromagnetics and Acoustics, EPFL. Since 1991, he has been a Professor with EPFL, and since 2000, he has been the Head of the Laboratory of Electromagnetics and Acoustics (LEMA), EPFL. In 1984, he was a Visiting Research Associate with the Rochester Institute of Technology, Rochester, NY, and Syracuse University, Syracuse, NY. He has also held scientific appointments with the University of Rennes, Rennes, France, the University of Nice, Nice, France, the Technical University of Denmark, Lyngby, Denmark, and the University of Colorado at Boulder. He is currently the Chairman of the EPFL Space Center and is responsible for many Swiss research projects for the European Space Agency (ESA). He has authored five book chapters on microstrip antennas and circuits and over 100 reviewed papers. His research interests include EM theory, numerical methods, and planar antennas.

Dr. Mosig has been a member of the Swiss Federal Commission for Space Applications. He is currently a member of the Board of the Applied Computational Electromagnetics Society (ACES), the chairman of the European COST Project on Antennas ASSIST (2007-2011), and a founding member and acting chair of the European Association and the European Conference on Antennas and Propagation (EurAAP and EuCAP). 